\title{
Avaliação do impacto de ambientes gamificados no processo de ensino-aprendizagem de programação de computadores: uma comparação entre elementos monousuário e multiusuário
}

\author{
Fabrício de S. Ribeiro', Bruno Merlin ${ }^{1}$, Heleno Fülber ${ }^{1}$ \\ ${ }^{1}$ Programa de Pós-graduação em Computação Aplicada \\ Universidade Federal do Pará (UFPA) - CEP 68464-000 - Tucurú́, PA - Brasil \\ fabricio.ribeiro@ifpa.edu.br, brunomerlin@ufpa.br, fulber@ufpa.br
}

\begin{abstract}
There are studies that aim to investigate solutions related to programming learning. One of the proposed alternatives is gamification. The elements of gamified environments based on digital games can be classified as single user or multi user. Thus, this study aims to evaluate the impact of gamified environments on the computer programming teaching-learning process by comparing these elements. For this purpose, a controlled experiment was conducted with a group of students. Among the results we could see the increase in student engagement after the insertion of multiuser elements in the gamified environment.
\end{abstract}

Resumo. Existem estudos que têm como objetivo a investigação de soluções relacionadas a aprendizagem de programação. Uma das alternativas propostas é a gamificação. Os elementos dos ambientes gamificados, baseados em jogos digitas, podem ser classificados em monousuário e multiusuário. Assim, este estudo tem o objetivo de avaliar o impacto de ambientes gamificados no processo de ensino-aprendizagem de programação de computadores através da comparação entre estes elementos. Com este propósito, foi realizado um experimento controlado com um grupo de alunos. Entre os resultados encontrados pôde-se observar o aumento no engajamento discente após a inserção de elementos multiusuário no ambiente gamificado.

\section{Introdução}

A aprendizagem de programação de computadores é fundamental em cursos da área de Tecnologia da Informação (TI). Afinal, este conhecimento objetiva ensinar como utilizar o computador para solucionar problemas do mundo real, através da construção de programas e sistemas computadorizados. Contudo, historicamente as disciplinas introdutórias aos conceitos de programação de computadores são desafiadoras para a maioria dos alunos. Conforme Neto e Schuvartz (2007), acadêmicos iniciantes, ao se depararem com a disciplina, sentem-se incapazes de programar devido ao conjunto de habilidades que a programação exige como capacidade para solucionar problemas, raciocínio lógico, habilidade matemática, capacidade de abstração, entre outras.

Desse modo, é necessário que sejam providas maneiras alternativas de auxiliar os discentes no processo de compreensão e aplicação dos conceitos de programação e 
VIII Congresso Brasileiro de Informática na Educação (CBIE 2019)

Anais do XXX Simpósio Brasileiro de Informática na Educação (SBIE 2019)

algoritmos. Um dos possíveis caminhos existentes na literatura é a Gamificação [DETERDING et al. 2011], no qual se busca motivar, engajar e, idealmente, modificar positivamente o comportamento de um determinado público-alvo, através do uso de recursos e elementos do mundo dos jogos. Para Savi e Ulbricht (2008), os jogos educacionais proporcionam práticas interativas e inovadoras, nas quais o aluno tem a chance de aprender de forma mais ativa.

Os jogos digitais vêm sendo cada vez mais utilizados para implementar as técnicas de gamificação. Há algum tempo estes também vêm ganhando espaço na utilização de recursos de telecomunicações, uma vez que esta tecnologia permite que os usuários consigam estabelecer interações com outros jogadores geograficamente distantes. Diversos estudos relatam a efetividade da gamificação, como o estudo de Penteado e Damasceno (2014), que alegam terem obtido sucesso na abordagem desse tema no ensino de programação de computadores.

Dentre as diversas dimensões, os jogos podem ser classificados em jogos para um jogador (monousuário), em que a execução prevê um único usuário como jogador, e os jogos para múltiplos usuários (multiusuário), em que a execução prevê dois ou mais usuários como participantes do tema desenvolvido no jogo. A utilização destes jogos, tanto aqueles com foco educacional quanto os de entretenimento, apresentam uma grande expansão no cenário mundial [PEIXOTO et al. 2015]. O estudo de Penteado e Damasceno (2014), assim como muitos trabalhos que abordam este assunto, é caracterizado pelo uso de um ambiente orientado a elementos monousuário.

O presente trabalho tem como objetivo avaliar o impacto de ambientes gamificados no processo de ensino-aprendizagem de programação de computadores através da comparação entre os elementos monousuário e multiusuário. Portanto, este artigo está organizado em cinco seções. A seção 2 expõe trabalhos relacionados ao presente estudo. De outro modo, a seção 3 apresenta a metodologia de pesquisa utilizada. Logo após, na seção 4, os resultados gerais. E, por fim, na seção 5, são feitas as considerações finais.

\section{Trabalhos Relacionados}

Iniciativas relacionadas ao tema gamificação no ensino-aprendizagem de fundamentos de programação de computadores vêm sendo realizadas com o fim de responder questões sobre o assunto. Neste sentido, pode-se observar o estudo de Zanchett et al. (2015), que buscou investigar a utilização de jogos de programar como uma forma de estabelecer os primeiros contatos de estudantes de ensino médio com conceitos de programação. No estudo, foram feitos experimentos com os jogos Code Combat $^{1}$, Lightbot ${ }^{2}$ e NoBug's Snack Bar ${ }^{3}$. Quanto a opinião dos estudantes participantes, os pesquisadores buscaram conhecer jogo preferido, engajamento e diversão, estilo tutorial e percepção sobre a programação e conceitos envolvidos. Segundo os autores, os resultados indicam que a abordagem do uso de jogos de

\footnotetext{
${ }^{1} \mathrm{https}: / /$ codecombat.com/

2 http://www.lightbot.com

${ }^{3}$ http://nobugssnackbar.dei.uc.pt/
} 
programação nos contatos iniciais dos estudantes pode trazer resultados benéficos ao criar ambientes em que o estudante possa atingir os objetivos educacionais enquanto se diverte.

Martins et al. (2016) descrevem em seu estudo um relato de experiência da aplicação do jogo Labirinto Clássico da plataforma Code.org ${ }^{4}$, com crianças do ensino fundamental. Os pesquisadores coletaram dados sobre a motivação, experiência do usuário e aprendizagem dos alunos. Segundo os autores, o estudo proporcionou uma ótima experiência aos usuários, alinhando a motivação e aprendizagem. De acordo com seus resultados, a maioria dos alunos não possuíam experiência prévia com os conceitos de programação, contudo, indicaram aumento em seu conhecimento sobre programação após o uso da ferramenta.

O estudo de Penteado e Damasceno (2014) discorre sobre o uso de jogos digitais no ensino de lógica de programação para o ensino médio integrado em informática. Ele apresenta uma forma alternativa de ensino da informática a partir de jogos digitais. $\mathrm{O}$ trabalho buscou identificar os benefícios e contribuições dos jogos digitais no ensino da informática, focando na disciplina de linguagem de programação. $\mathrm{O}$ trabalho utilizou $\mathrm{O}$ jogo Code Combat para verificar se este possui as habilidades necessárias no auxílio da disciplina. De acordo com os resultados coletados pelos autores, há existências dos benefícios e contribuições sobre o tema tratado, e constataram que o recurso utilizado pode ser aplicado em tarefas nas quais a teoria deve ser aliada à prática.

O trabalho de Pantaleão et al. (2017), sobre o uso da ferramenta Robocode ${ }^{5}$ no ensino de algoritmos e programação de computadores para alunos do ensino médio, investiga uma abordagem para inserção da programação no ensino médio como forma de despertar e/ou fortalecer o raciocínio matemático a dos estudantes. Para este fim, os pesquisadores utilizaram o ambiente Robocode como ferramenta de apoio ao ensino da linguagem de programação Java, visando apresentar conceitos básicos de programação para os alunos de forma rápida e divertida. A partir dos resultados obtidos, os pesquisadores acreditam que por meio deste tipo de metodologia venha a ser desenvolvido o senso crítico dos alunos envolvidos, estimulando a busca por conhecimento e competição. Para os autores, através da realização dos torneios de Robocode, foi possível observar a influência destes na motivação dos estudantes em aprender e superar desafios.

É possível observar nos trabalhos citados, a presença de similaridades quanto aos objetivos deste estudo. Os autores buscaram avaliar o impacto do uso de ambientes gamificados no processo de ensino-aprendizagem dos conceitos de programação de computadores, mas não incluíram em seus objetivos ou nos resultados encontrados, a influência dos aspectos dos ambientes relacionados aos elementos monousuário e multiusuário. Logo, faz-se necessário avaliar a influência desses elementos no processo de ensino-aprendizagem de programação de computadores.

\footnotetext{
${ }^{4} \mathrm{https}$ ://studio.code.org/s/hourofcode

${ }^{5} \mathrm{https}$ ://robocode.sourceforge.io/
} 
VIII Congresso Brasileiro de Informática na Educação (CBIE 2019)

Anais do XXX Simpósio Brasileiro de Informática na Educação (SBIE 2019)

\section{Metodologia}

No intuito de avaliar os benefícios dos aspectos multiusuário, a metodologia se baseou na seleção e alteração de um ambiente gamificado de forma que o ambiente possa ser explorado em modo monousuário ou multiusuário, e na realização de um experimento comparativo envolvendo os dois modos de uso.

\subsection{Definição do ambiente gamificado}

A maioria das aplicações que empregam conceitos de gamificação estabelece alguma forma de recompensa pelas atividades realizadas pelo usuário. Existe uma série de elementos de design de jogos que podem ser incorporados: avatares, recursos colecionáveis, recompensas, níveis (sistema de progressão), desbloqueio de conteúdo, combates e outros. No entanto, segundo Werbach e Hunter (2012), os elementos de jogos mais utilizados correspondem ao trio PBL (do acrônimo em inglês PBL - points, badges, leaderboards), que são mecanismos básico de um game com a finalidade de promover mudanças no comportamento dos indivíduos:

- Pontos (pontuação que o jogador recebe ao executar determinadas ações ou comportamentos incentivados pelo processo);

- Medalhas (marcos ou conquistas que são dados ao jogador como uma forma de reconhecer que ele atingiu um patamar ou status desejado);

- Ranking de Líderes (classificação do jogador com relação aos outros jogadores envolvidos no processo, usada como métrica de comparação de sua performance).

Para a realização do experimento era necessário o uso de um ambiente que tenha como características a implementação de todos os elementos propostos pela PBL, monousuário e multiusuário. Neste contexto, os elementos monousuário podem ser pontuação, títulos, status e outras recompensas que são restritos ao próprio usuário. Por outro lado, o principal recurso multiusuário tratado na PBL é ranking, este recurso mostra as informações, como pontuação, dos demais usuários de um grupo e permite eles determinarem sua posição perante os outros, eles são uma forma de feedback específico sobre a competição [DUARTE 2019].

Os jogos digitais voltados para o ensino-aprendizagem dos conceitos de programação encontrados literatura foram: Robocode, Code Combat, Labirinto Clássico do Code.org, Scratch, Lightbot, SpriteBox e NoBug's Snack Bar. Contudo, observou-se que nenhum deles faz uso do PBL em sua completude, a maioria destes possui elementos voltados a um único usuário (monousuário), deixando de lado recursos multiusuário.

Portanto, foi necessário realizar modificações em um dos jogos digitais listados para agregar os recursos ausentes para ser realizado o experimento proposto pelo estudo. O Code Combat foi escolhido em razão de possuir código open-source, já possuir a maioria dos elementos do PBL e possuir uma comunidade participativa. É importante frisar que o Code Combat possui elementos adicionais que ultrapassam o escopo da PBL, como gemas, armas, mascotes e outros. 
VIII Congresso Brasileiro de Informática na Educação (CBIE 2019)

Anais do XXX Simpósio Brasileiro de Informática na Educação (SBIE 2019)

O Code Combat é um jogo que aborda conceitos de programação através de uma aventura medieval, onde o jogador controla heróis, e ao longo de sua jornada vai coletando cristais e derrotando diversos inimigos, como ogros, bandidos, entre outros [CODECOMBAT 2019]. O jogo disponibiliza um ambiente de programação próprio para que o jogador implemente seus códigos. Este ambiente trabalha com 3 linguagens de programação, sendo elas: Python, JavaScript e CoffeeScript (Experimental). A Figura 1 apresenta a primeira missão do curso Introdução à Ciência da Computação. $O$ personagem necessita concluir todos os objetivos para completar missão. Ele se locomove no ambiente de acordo com o algoritmo definido no editor de código. A missão contextualizada na figura abaixo faz a abordagem da sintaxe básica do jogo.

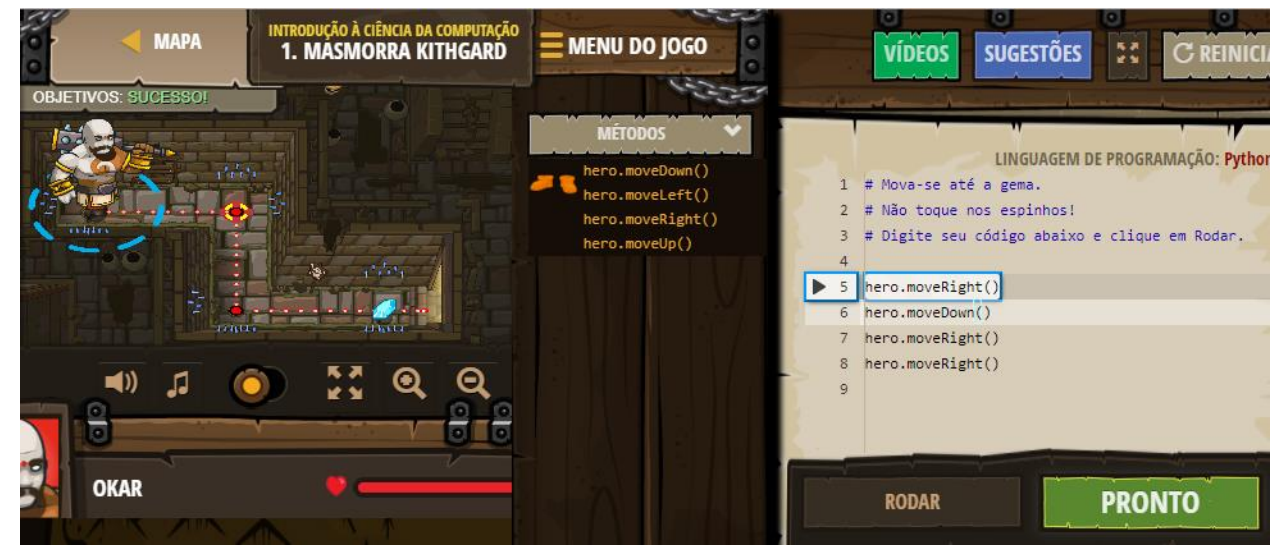

Figura 1 - Demonstração do jogo.

Fonte: www.codecombat.com

O ranking padrão dos jogos digitais convencionais permite aos jogadores acompanharem o progresso dos outros usuários durante todo o tempo de jogo. Em razão disso, foi necessário desenvolver esta versão do ranking para ser integrada ao Code Combat para, assim, este se alinhar ao PBL. O ranking desenvolvido apresenta o progresso de todos os alunos da turma quanto ao avanço em determinado curso. Esse recurso torna-se disponível desde a realização da primeira missão. Esta versão do ranking foi enviada ao servidor do jogo e integrada a este.

\subsection{Avaliação}

A avaliação foi dividida em 2 fases: Experimentação (contato dos estudantes com o Code Combat); e teste de retenção de conhecimento (teste sobre conceitos de programação de computadores a fim verificar o grau de assimilação do conteúdo abordado). Na primeira fase foram utilizados observações e dados extraídos do ambiente gamificado como recursos de coleta de dados. Na segunda, por outro lado, foram utilizados questionários.

\subsubsection{Experimentação}

Tendo como norte os objetivos do estudo, o experimento contou com participação de dois grupos de estudantes que fizeram uso do Code Combat. O primeiro grupo utilizou recursos do jogo correspondentes aos elementos monousuário (pontos e medalhas do PBL). O segundo grupo teve acesso a todos os recursos do jogo 
VIII Congresso Brasileiro de Informática na Educação (CBIE 2019)

Anais do XXX Simpósio Brasileiro de Informática na Educação (SBIE 2019)

correspondentes aos elementos monousuário e multiusuário (ponto, medalhas e ranking de líderes do PBL).

As atividades de experimentação foram realizadas durante o período de abril a maio de 2019. A amostra foi extraída a partir da turma do primeiro ano do curso Técnico em Desenvolvimento de Sistemas integrado ao Ensino Médio do Instituto Federal de Educação, Ciência e Tecnologia do Pará (IFPA) - Campus Óbidos-PA. Até a realização do experimento, os alunos não haviam tido contato com a programação de computadores. A amostra foi classificada em dois grupos: um grupo de controle, utilizando somente os elementos monousuário - grupo 1 (19 alunos); e um grupo experimental, usando todos os recursos, monousuário e multiusuário - grupo 2 (19 alunos). A definição dos membros de cada grupo foi dada a partir de sorteio. Os grupos (grupo de controle e grupo experimental) foram separados a fim evitar interferência da relação destes no resultado. Para participação na atividade, os estudantes receberam um Termo de Consentimento Livre e Esclarecido (TCLE) para que pudessem solicitar a assinatura de seus responsáveis.

O experimento foi executado por meio da oferta de um minicurso de introdução a programação de computadores, com carga horária de 10 horas e 40 minutos, distribuídas em 3 (três) semanas. Cada grupo experimental contou com 6 (seis) encontros presencias. Ambos os grupos receberam orientações iniciais para o jogo, material impresso de apoio e um conjunto de exercícios. O material de apoio e exercícios foram adaptados a partir do conteúdo disponível no site oficial do Code Combat. Tiveram acompanhamento de um professor e 3 alunos voluntários para auxiliálos na solução de eventuais dúvidas. Após as orientações iniciais do primeiro encontro, cada aluno progrediu no jogo de acordo com seu ritmo. Desse modo, os estudantes não foram compelidos a progredir no jogo por influência de uma autoridade presente no laboratório de informática.

Foram disponibilizados aos grupos dois cursos disponíveis no site do jogo: Introdução à Ciência da Computação e Ciência da Computação 2. Cada curso possui um mapa diferente: o primeiro é composto por 38 missões, estas abordam sobre conteúdos relacionados a sintaxe básica, laços de repetição e variáveis; o segundo curso foi liberado para cada aluno após a conclusão do primeiro, é composto por 73 missões, aborda sobre condições se/senão, condições aninhadas, funções e eventos.

Os estudantes do grupo 2 (G2) foram apresentados, ainda no primeiro encontro, ao ranking da turma (Figura 2), que apresenta a classificação de todos os jogadores do grupo. A classificação é realizada a partir do progresso individual de cada estudante, ou seja, conforme o estudante avança na conclusão das missões ele consegue 1 (um) ponto no ranking. O desempate é realizado pelo menor tempo de solução. Quanto maior é o número de missões (níveis) concluídos por um aluno e menor for o tempo de conclusão, melhor será sua classificação. A quantidade de missões concluídas é representada nesta imagem pelo termo "Níveis Completos". O ranking foi desenvolvido para os dois mapas disponíveis para o $\mathrm{G} 2$. 
VIII Congresso Brasileiro de Informática na Educação (CBIE 2019)

Anais do XXX Simpósio Brasileiro de Informática na Educação (SBIE 2019)

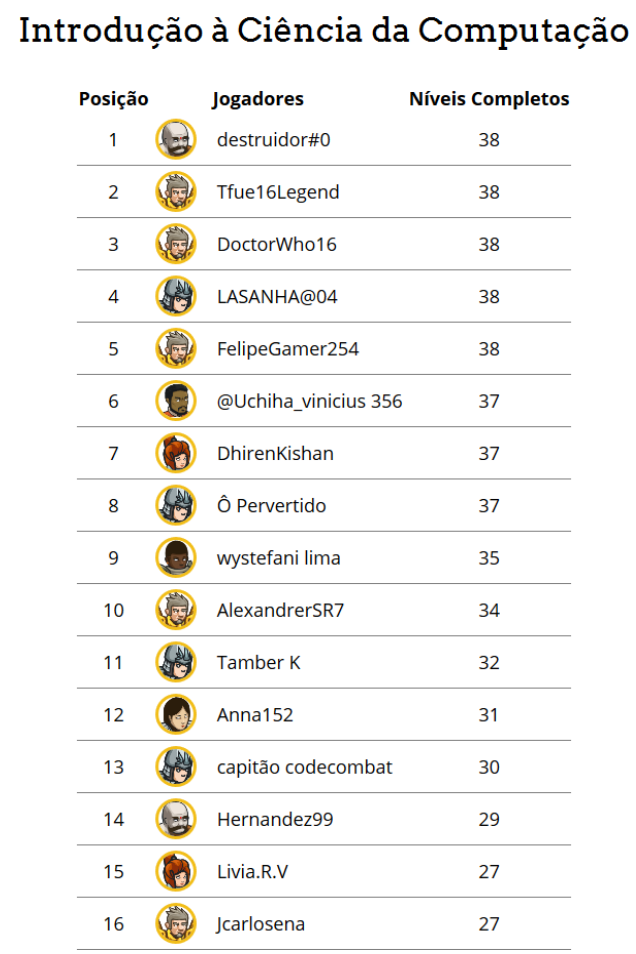

Figura 2 - Ranking desenvolvido para o curso Introdução à Ciência da Computação do G2.

\subsubsection{Teste de retenção de conhecimento}

Para avaliar o desempenho dos discentes, quanto à aprendizagem de programação de computadores através do uso do ambiente gamificado, foram aplicados questionários através de um pós-teste. O questionário aplicado é composto por 11 (onze) perguntas que tratam de conceitos abordados no primeiro mapa do jogo, com a finalidade de aferir a absorção dos discentes quanto aos conceitos sobre a sintaxe básica, laços de repetição e variáveis. Alguns alunos não alcançaram as missões do segundo mapa, em virtude disso, os conceitos relacionados ao segundo mapa (condições se/senão, condições aninhadas, funções e eventos) não compuseram este questionário.

\subsubsection{Instrumento para aferir o engajamento}

A fim de aferir a dedicação, participação e frequência de uso da ferramenta foram extraídos do Code Combat registros correspondentes: frequência de acesso a ferramenta; tempo de jogo e quantidade de missões concluídas. Estes dados foram utilizados para aferir o engajamento dos estudantes quanto ao uso do jogo.

\section{Resultados Gerais}

Durante a realização dos experimentos controlados houve 3 (três) estudantes que deixaram de frequentar os encontros presencias e não realizaram acessos posteriores ao jogo. Esses alunos foram classificados como "evadidos", portanto seus dados não foram computados em seus respectivos grupos. Entre esses alunos que abandonaram o minicurso, 2 (dois) faziam parte do G1 e 1 (um) integrava o G2. Em razão disso, a 
VIII Congresso Brasileiro de Informática na Educação (CBIE 2019)

Anais do XXX Simpósio Brasileiro de Informática na Educação (SBIE 2019)

amostra real computada para o G1 foi 17 (dezessete) estudantes e para o G2 18 (dezoito). Portanto, reduzindo para 35 (trinta e cinco) alunos para a amostra geral.

Após a coleta dos dados, foi feita uma verificação da completude dos questionários respondidos durante a realização do pós-teste. Os dados individuais dos discentes também foram extraídos dos registros feitos pelo Code Combat. Os dados foram analisados para obtenção dos resultados da avaliação, apresentada nesta seção.

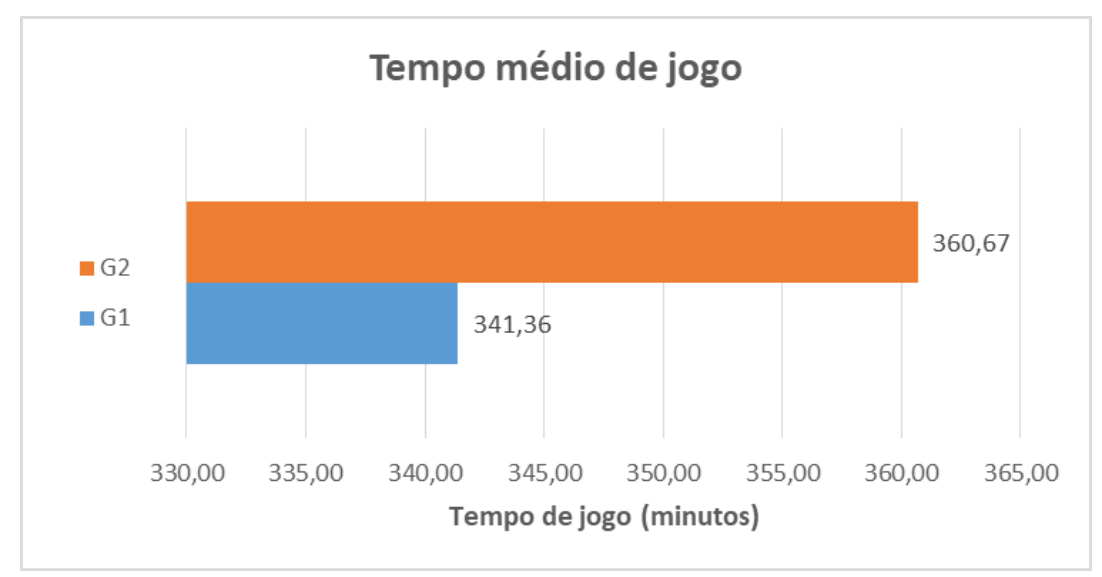

Figura 3 - Resultados sobre o tempo médio de acesso ao jogo.

A Figura 3 apresenta o gráfico de tempo médio de jogo. Pode-se observar que os alunos do G2 permaneceram em média 360,67 minutos conectados ao jogo, enquanto os estudantes do G1 estiveram em média 341,36 minutos durante o período do experimento. Estes dados mostram 5,66\% de diferença de tempo entre os grupos.

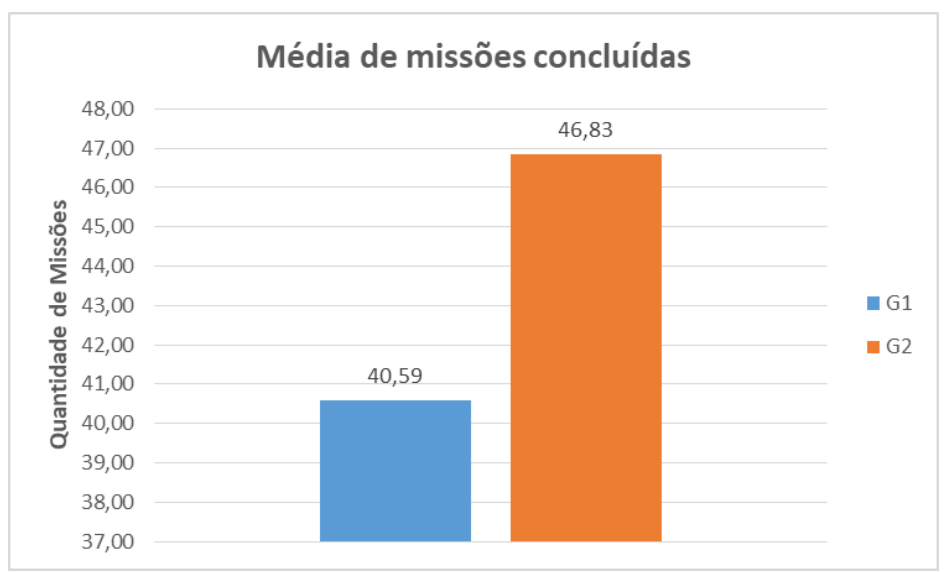

Figura 4 - Resultado sobre a quantidade média de missões concluídas.

A figura anterior deixa evidente que os alunos do G2 realizaram mais missões do Code Combat que os alunos do G1. Vale observar que a soma do quantitativo de missões entre o primeiro e o segundo mapa corresponde a 111 (cento e onze). A figura anterior mostra uma discrepância de 15,4\% de missões completas entre o G1 e G2.

A análise do teste de retenção de conhecimento, ou pós-teste, não revelou diferença significativa entre os acertos dos alunos dos grupos, pois através do teste de hipóteses t de Student pode-se observar que o $t$ cálculo $=1,37$ é menor que o $t$ crítico $=$ 2,08 e/ou $p$-valor $=0,18(18 \%)$ é maior que $\alpha(0,05$ ou $5 \%)$. Em razão disso, pode-se 
VIII Congresso Brasileiro de Informática na Educação (CBIE 2019)

Anais do XXX Simpósio Brasileiro de Informática na Educação (SBIE 2019)

concluir que não houve diferença de abstração dos conceitos de programação abordados no primeiro mapa do jogo.

Os gráficos das figuras 3 e 4 realçam a influência do ranking desenvolvido no espírito de competividade entre os estudantes, em consequência, isto resultou no aumento do engajamento dos discentes do G2 sobre dos G1, ou seja, aumento número de tempo de jogo e missões concluídas.

Pode-se observar ao longo da realização do experimento, com ambos os grupos, que os alunos demonstravam satisfação, alguns por estarem se divertindo, outros por estarem se divertindo enquanto aprendiam programação. Em relação aos alunos do G2, foi possível presenciar, em vários momentos distintos, estudantes perguntando aos seus colegas sobre sua atual posição no ranking, na expectativa de saber se conseguiu superálos ou distanciar em número de conclusão de missões.

\section{Conclusões e Trabalhos Futuros}

Através do desenvolvimento deste trabalho, pode-se observar, na prática, os benefícios descritos nos resultados de estudos que examinaram a aplicação da gamificação no ensino-aprendizagem de programação de computadores, como no relato de Zanchett et al. (2015), quando descrevem que os objetivos educacionais podem ser atingidos enquanto os estudantes se divertem. É importante frisar que esses estudos tiveram como alicerce de suas afirmações dados extraídos de ambientes gamifiados que possuem, prevalecentemente, elementos monousuário.

O experimento também alinha-se com o estudo de Penteado e Damasceno (2014), uma vez pode-se concluir que de fato a gamificação tras benefícios e contribuições no ensino-aprendizagem de programação de computadores. Um dos benefícios observados que se pode citar é o entusiasmo dos discentes ao fazer uso de um recurso metodológico e lúdico que está fortemente relacionado ao contexto extraclasse que muitos participam.

Diante dos resultados apresentados, pode-se concluir que após a adição dos elementos multiusuário, ranking de líderes, no Code Combat, houve aumento no engajamento dos discentes em relação ao ambiente gamificado, portanto acréssimo no tempo de jogo e quantidade de missões completas. Através da análise dos dados obtidos, não é plausível afirmar, mas deduzir, que o aumento do engajamento refletiu no índice de abandono dos grupos, uma vez que o grupo que não fez uso dos recursos multiusuário apresentou maior número de alunos evadidos, levando-nos a especular a relação entre o engajamento e a evazão discente.

Foi observado também que o aumento do engajamento dos discentes não implica necessariamente no aumento significativo da abstração dos conceitos abordados pelo ambiente gamificado. Assim faz-se necessário um intermediador para revisar e exercitar o tema estudado, de modo que venha maximizar a experiência e os objetivos educacionais.

Sugestões de trabalhos futuros incluem a realização de novos experimentos agregando novos recursos multiusuário, como elementos de colaboração (participação de clãs ou alianças) e eventos de competição (torneios). O foco estará em elucidar mais 
VIII Congresso Brasileiro de Informática na Educação (CBIE 2019)

Anais do XXX Simpósio Brasileiro de Informática na Educação (SBIE 2019)

profundamente as característicacas dos elementos multiusuário e seu impacto no engajamento e abstração dos conceitos abordados.

\section{Agradecimento}

Os autores agradecem a startup CodeCombat Inc, responsável pelo jogo Code Combat, que através de seu Chief Executive Officer (CEO), Nick Winter, e sua equipe de engenheiros, disponibilizou: suporte técnico durante as modificações desenvolvidas no ambiente; hospedagem destas em seus servidores; e licenças para acesso aos cursos utilizados por todos os envolvidos no experimento.

\section{Referências}

CODECOMBAT. Sobre o CodeCombat. Disponível em www.codecombat.com/about. Acesso em 01/07/2019.

DETERDING, S., DIXON, D., KHALED, R., NACKE, L. (2011). From game design elementsto gamefulness: Defining "gamification". In.: Proceedings of the 15th International Academic MindTrek Conference: Envisioning Future Media Environments, MindTrek '11, New York, NY, USA. ACM.

DUARTE, P. Gamificação. Disponível em https://letscodeacademy.com/blog/gamificacao/. Acesso em 02/07/2019.

MARTINS, R.; REIS, R; MARQUES, A. Inserção da programação no ensino fundamental Uma análise do jogo Labirinto Clássico da Code.org através de um modelo de avaliação de jogos educacionais. In.: Workshop de Informática na Escola, 2016, Porto Alegre.

NETO, W. C. B.; SCHUVARTZ, Aguinaldo. Ferramenta computacional de apoio ao processo de ensino-aprendizagem dos fundamentos de programação de computadores. In: SBIE 2007, São Paulo. Anais.

PANTELÃO, E.; AMARAL, L.; SILVA, G. Uma abordagem baseada no ambiente Robocode para ensino de programação no Ensino Médio. In.: Revista Brasileira de Informática na Educação, 2017, São Paulo.

PEIXOTO, Daniela C. C.; SANTOS, Silas T.; RESENDE, Rodolfo F. Avaliação de Jogos Digitais Educacionais Multiusuários: Uma Revisão Sistemática da Literatura. In: Simpósio Brasileiro de Informática na Educação - SBIE, v. 26, 2015. Anais.

PENTEADO, C.; DAMASCENO, E. Contribuições de jogos digitais no ensino da lógica de programação para o ensino médio integrado em informática. Revista E.T.C. Logomarca. Edição 01. 2014.

SAVI, R.; ULBRICHT, V. R. (2008) "Jogos Digitais Educacionais: Benefícios e Desafios.", Revista Novas Tecnologias na Educação, 6, p. 1-10.

WERBACH, K.; HUNTER, D. For the win: how game thinking can revolutionize your business. Philadelphia: Wharton Digital Press, 2012.

ZANCHETT, G.; VAHLDICK, A.; RAABE, A. Jogos de Programar como uma Abordagem para os Primeiros Contatos dos Estudantes com a Programação. In.: Workshop do Congresso Brasileiro de Informática na Educação, 2015, Recife. 